\title{
Step Line Tension on a Metal Electrode
}

\author{
H. Ibach \\ Institut für Schichten und Grenzflächen, ISG3 Forschungszentrum Jülich, D-52425 Jülich, Germany
}

W. Schmickler*

Abteilung Elektrochemie, University of Ulm, D-89069 Ulm, Germany

(Received 26 February 2003; published 3 July 2003)

\begin{abstract}
The step line tension in electrochemical systems differs conceptually from the line tension on metals in the vacuum because it refers to different boundary conditions. A procedure is established for calculating the electrochemical line tension and is applied to a novel model of the interface comprising both a stepped metal electrode and an electrolyte solution. To first order, the potential dependence of the line tension is governed by the energy of the step dipole in the electric field of the space charge in the solution.
\end{abstract}

DOI: 10.1103/PhysRevLett.91.016106

PACS numbers: 68.08.De, 68.35.Md

The line tension $\beta$ of a two-dimensional metal island governs it shape, its growth and decay, and it thus plays a crucial role in the electrochemical deposition and dissolution of metals, and, in particular, in the creation of metallic nanostructures. However, despite its importance, there have been very few investigations of the line tension in electrochemical systems. Experimentally, this quantity is difficult to measure: Until very recently, it could be determined only from the critical cluster size of islands on perfect single-crystal electrodes, and the only values available were those for silver islands on electrodes grown in capillary tubes [1]. In addition, these investigations were limited to a very small range of electrode potentials $\phi$ a little below the thermodynamic deposition potential, so that nothing was known about the potential dependence of $\beta$. Because of the paucity of experimental data, the line tension and its dependence on electrochemical quantities was disregarded by theorists.

During the last few years the situation has changed, and a new method has been developed in which the line tension is obtained from the fluctuations of the island perimeter observed with a scanning tunneling microscope [2-4]. Stimulated by these experimental achievements we have investigated the nature of the step line tension for metals in contact with an electrolyte theoretically, and present the first model for the step line tension and its dependence on the electrode potential which comprises both the metal and the electrolyte. The contribution of the electrolyte alone to the line tension, without accounting for the solid state aspects, was considered in [5]. We will focus on the basic case in which specific adsorption is absent and present a simple model from which basic trends can be deduced. We find that the dominant contribution to the potential dependence of the line tension arises from the energy of the step dipole moment in the electric field of the double layer. To first order, this gives rise to a linear dependence of the line tension on the electrode potential. We argue that this result can be generalized to other defect energies as well as to activation energies for diffusion and coarsening processes.

Before presenting our model, we note that there is a crucial difference between the line tension in electrochemistry and the same quantity in surface science. In electrochemistry the experiments are conducted at a constant electrode potential. Fluctuations in the island perimeter involve changes in the surface dipole potential, since the edges carry an excess dipole moment. Therefore, in order to keep the electrode potential constant, edge fluctuations must involve small fluctuations of the charge density $\sigma$ in the vicinity of the edge. In contrast, experiments in surface science are conducted at zero excess charge, so that island fluctuations are accompanied by fluctuations of the local work function. This implies that different thermodynamic potentials need be invoked for the description of surfaces in vacuum and in contact with an electrolyte. We will illustrate this point in our calculations below.

In order to gain further insight into the electrochemical line tension we have performed explicit model calculations for the basic case in which specific adsorption of ions plays no role. In the construction of the model we have been guided by the theory of the electric double layer, where a model of jellium in contact with a spacecharge region has helped greatly in our understanding of the interface [6]. Therefore, we represent the metal as jellium with a lattice of pseudopotentials (see Fig. 1) with an additional monatomic terrace. The introduction of pseudopotentials is necessary since simple jellium has a pathological surface energy for higher electronic densities. We have followed the work of Lang and Kohn [7] and employed Ashcroft potentials [8], which were averaged parallel to the surface, except for the edges, where the pseudopotentials of the edge atoms were averaged parallel both to the surface and to the edge. Periodic 


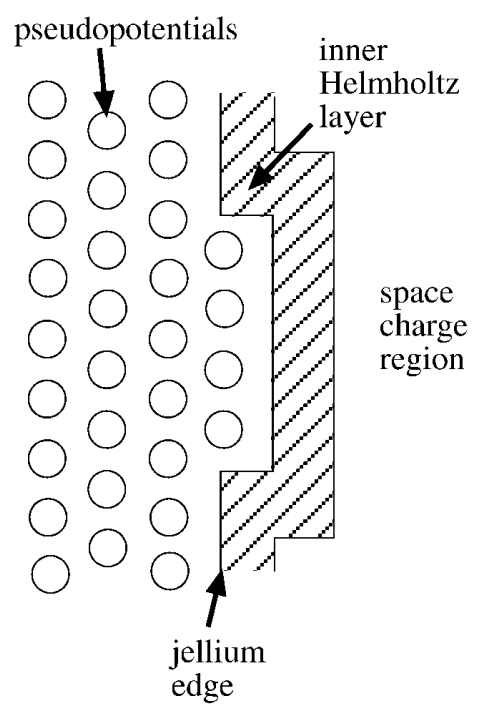

FIG. 1. Model for calculating the line tension of a metal island in an electrochemical environment. The diagram shows a cross section; the electrode, with an island on top, is to the left and the solution to the right.

boundary conditions apply in the $x$ and $y$ directions parallel to the surface. Adjacent to the surface is an outer Helmholtz layer [9], into which the ions cannot penetrate, and where the effective dielectric constant is lowered; this we have modeled as a region with a dielectric constant of $\epsilon=1$ and a thickness of 5 a.u. This effective dielectric constant is lower than the value of $\epsilon=5-6$ that is usually ascribed to the Helmholtz layer in aqueous solutions. Consequently the calculated interfacial capacities are lower than those that are typically found for aqueous solutions and correspond more to nonaqueous solvents. However, a model with three different dielectric constants (metal, Helmholtz layer, space-charge region) would make the calculations more complex, without providing deeper insight into the physics of the system. The space-charge region beyond the Helmholtz layer was treated by the linear Poisson-Boltzmann theory, assuming a dielectric constant of 80 and a Debye length of 10 a.u. Using the linear version of the Gouy-Chapman theory not only simplifies the calculations, but is justified because the Helmholtz layer reduces the electric field significantly. For a nonlinear treatment see [5]. Here we focus on the metal, and this simple model of the electrolytic double layer suffices for our purposes. The same model has been successfully used by Schmickler and Leiva [10] in an investigation of the surface tension and stress of metal electrodes.

The jellium and the space-charge region interact electrostatically. In the calculations this is achieved by solving a differential equation which reduces to the Poisson equation in the jellium and to the Poisson-Boltzmann equation in the space-charge region.

For jellium calculations, the charge density $\sigma$ is the natural variable - the transformation to constant elec- trode potential has to be done later. The equilibrium configuration for a given value of $\sigma$ is obtained by minimizing the free energy of the system, which consists of the internal energy of the jellium (which can be taken at $T=0$ ) and the free energy stored in the electrostatic field. For this purpose, we have parametrized the electronic density through a family of trial functions with three free parameters, using Smith-type exponentials [11] both at the jellium edge and at the edge of the island. For a flat surface and zero excess charge, our parametrization reduces to that used by Smith. The free parameters were then determined by minimizing the free energy of the system. Our approximate results compare well with the numerically exact results of Ishida and Liebsch [12], and compare favorably with the parametrization of Thompson and Huntington [13]. In any case, our model is good
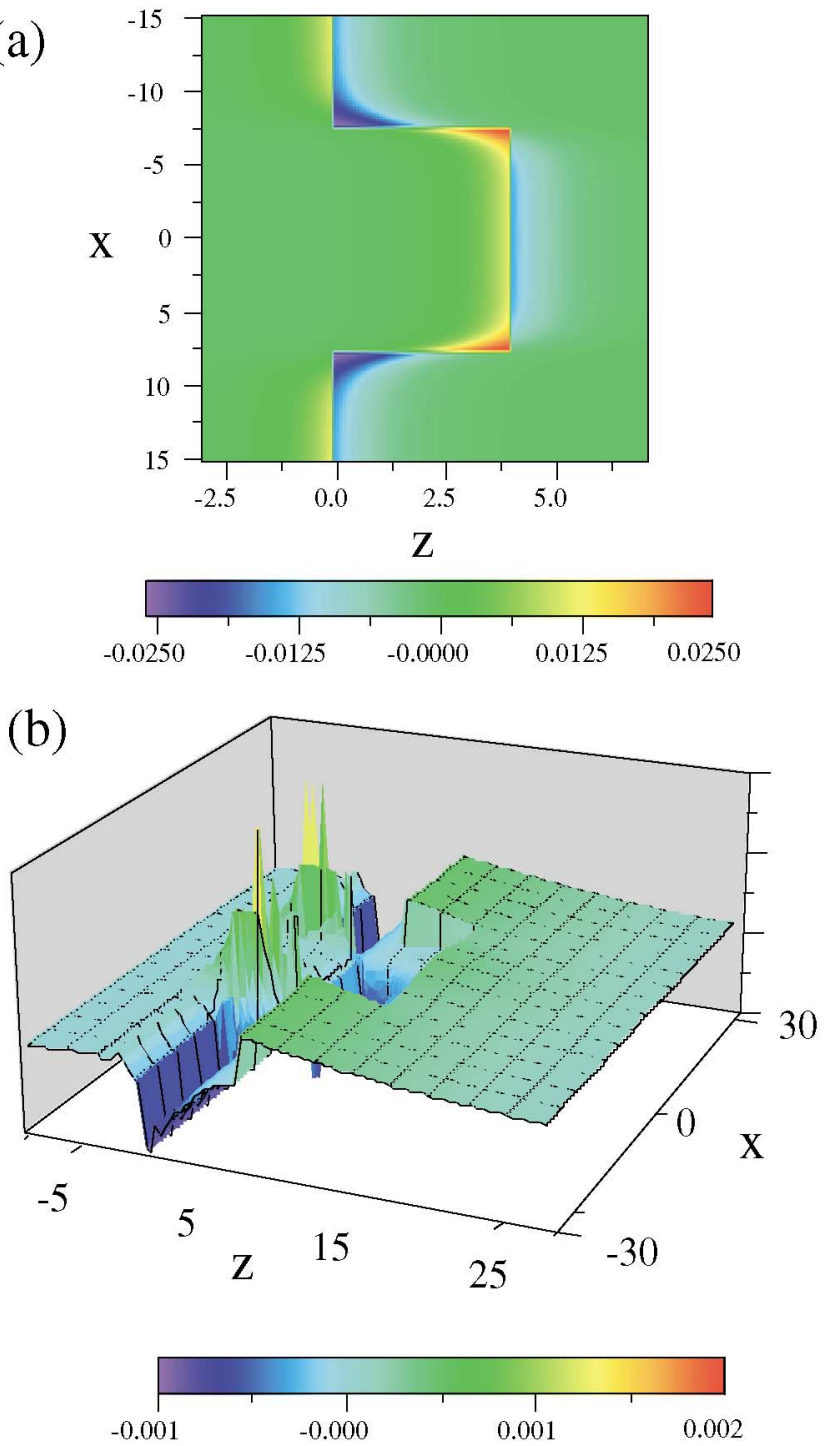

FIG. 2 (color). (a) Charge distribution at the potential of zero charge. (b) Distribution of the excess charge for a negatively charged electrode. The color code gives the charge density in atomic units. 
enough to gain a basic understanding of the edge energy, as we shall see below.

We have chosen $\mathrm{Al}(100)$ as our model system taking an Ashcroft radius of $r_{s}=1.12$ (all values are given in atomic units, unless stated otherwise). The system size was 60 a.u. in the two directions parallel to the surface, and 70 a.u. perpendicular to the surface. The width of the monatomic terrace was 15 a.u., which is sufficiently wide so that the two edges do not interfere noticeably.

These calculations give the charge distribution and the free interfacial energy of the system in dependence of the average charge density $\sigma$ of the electrode. Because of the Smoluchowski effect [14] there is a dipole moment at the edges, the positive end pointing towards the solution [see Fig. 2(a)]; this leads to a reduction of the local work function, an effect familiar from stepped surfaces [15]. This dipole moment polarizes the adjacent solution, but this effect is too small to be visible in the presence of the large edge dipole. Figure 2(b) shows the excess charge distribution for a negative charge on the interface. Two opposing charge layers, one on the metal, the other in the solution, are clearly visible, as is the disturbance caused by the dipole moments at the step edges. The decay length for the charge distribution in the solution is given by the Debye length and is considerably larger than the decay length in the metal, which is of the order of 1 a.u. The large excesses at the edge show that the edge dipole possesses a noticeable polarizability; we shall see below that this affects the interfacial capacity.

The free interfacial energy of the system contains three terms: (1) the classical energy required to split the crystal, while keeping the electronic density frozen, i.e., steplike at the surface; (2) the energy change when the electronic density is relaxed to its real form; (3) the electrostatic free energy stored in the Gouy-Chapman region. Only the latter two are obtained from energy minimization; the former has to be calculated separately from classical electrostatics. For the flat surface, the classical splitting energy has been given by Lang and Kohn [7]. For the stepped surface, there is an additional term: a little thought shows that this is the energy required to split a single plane of atoms into two parts; this can be calculated by the same method as the splitting of a three-dimensional crystal. Within the jellium model, the step has a positive line tension if it is oriented along the $\langle 100\rangle$ direction, but not in the $\langle 110\rangle$ direction. Therefore, we have taken the step edge along the $\langle 100\rangle$ direction, so that the island is stable. Of course, in real metals the step energy is positive for either direction.

Figure 3 shows the free energy $F(\sigma)$ per unit area of the system as a function of the average surface charge density $\sigma$ both with the step and without. As expected, the stepped surface has the higher energy. However, as pointed out before, the experimental situation corresponds to a constant electrode potential, or to a constant electrochemical potential $\tilde{\mu}$. Therefore, for both systems, we have obtained the electrochemical potential from the

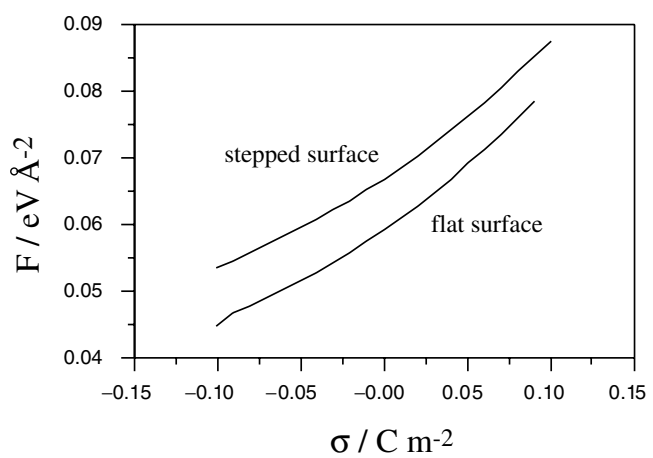

FIG. 3. Free energy of the stepped surface (upper curve) and a flat surface (lower curve) as a function of the surface charge density.

relation [10]

$$
-\tilde{\mu} / e_{0}=\frac{\partial F}{\partial \sigma}
$$

and then performed a Legendre transformation to obtain the surface tension $\gamma$ :

$$
\gamma=F-\sigma \tilde{\mu} / e_{0} .
$$

The results are shown in Fig. 4. The surface tensions both for the flat and for the stepped surfaces have the shape familiar from the electrocapillary equation: the surface tension attains its maximum at the potential of zero charge - this is a trivial consequence of the transformation (2). Since the stepped surface has a lower work function, its potential of zero charge is shifted to a lower value; in this case the shift is $0.14 \mathrm{eV}$. The capacity of the
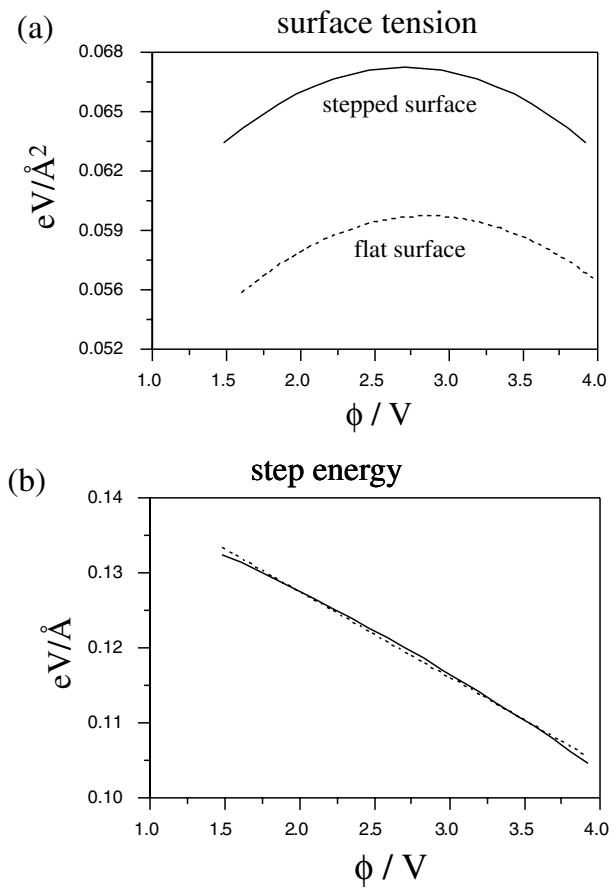

FIG. 4. (a) Surface tension for a stepped and a flat surface. (b) Step energy as a function of the electrode potential. The dashed line is the prediction of Eq. (3). 
interface can be obtained from the second derivative of the surface tension at the maximum. Since the dipoles at the step edges are polarizable, the capacity for the stepped surface is somewhat higher than that of the flat surface: $8.2 \mu \mathrm{F} \mathrm{cm}^{-2}$ as compared to $8.0 \mu \mathrm{F} \mathrm{cm}^{-2}$. In contrast, the contribution of the space-charge region to the line capacity is negative [5]. The step line tension is obtained as the difference between the two surface tensions, normalized per unit length. Since the stepped surface has a higher surface energy, the line tension $\beta$ is always positive over the investigated range — indeed, a negative line tension would lead to a spontaneous roughening of the surface. Since the potential of zero charge for the stepped surface is higher, and its capacity lower, the line tension decreases with the potential.

In a recent communication [5] Ibach et al. considered the contribution of the solution to the line tension and argued that the leading term in the potential dependence is simply given by the electrostatic energy of the edge dipole $p$ in the electric field of the double layer. Expressing the edge dipole moment through the change in work function, and relating the charge to the potential through the capacity, gives

$$
\frac{d \beta}{d \phi}=\frac{d}{d \phi}\{-4 \pi \sigma p / a\}=-4 \pi L \Delta \Phi C,
$$

where $L$ is the average width of the terrace, assuming equidistant line edges; $\Delta \Phi$ is the change in the work function due to the steps, $C$ is the interfacial capacity, which is assumed to be unchanged, and $a$ is the line length per atom. Substituting the values of our calculation gives an estimate of about $-1.1 \times 10^{-2} \mathrm{eV} \AA^{-1} \mathrm{~V}^{-1}$, which is the same as the initial slope of the line tension in Fig. 4(b). We note, however, that our calculations extend over a comparatively small range of $-0.1 \leq \sigma \leq 0.1 \mathrm{Cm}^{-2}$ of charge densities only, even though the potential range is quite large. This is a consequence of the low dielectric constant that we assumed for the Helmholtz layer, which results in a low interfacial capacity. Under the usual experimental conditions the capacities in aqueous solutions are substantially higher, so that much higher charge densities and fields can be reached. Under these circumstances nonlinear effects, such as those caused by the polarizability of the step dipole, will be larger than in our calculations. Nevertheless, it is pleasing that the general, first order relation (3) is verified by our explicit model calculations.

This agreement proves that the prevailing contribution to the potential dependence of the line tension is the energy of the edge dipole in the field of the double layer. This result has far-reaching consequences as it should be pertinent also to the potential dependence of other defect energies as well as to activation energies involved in atom migration. According to this model, defect formation energies and activation energies for migration should depend linearly on the electrode potential to lowest order, with a slope given by the dipole moment of the defect, and by the difference in the dipole moments in the transition state and the ground state, respectively. This provides a theoretical base for a successful heuristic ansatz which was made to describe the potential dependence of step fluctuations on several metal surfaces [16].

A comparison of our results with experimental values is not possible at this time because the only existing data are for gold islands in a sulphate solution, where the anion is known to adsorb strongly on the electrode. However, we expect that the qualitative results of our work: a decrease of the potential of zero charge, a concomitant shift of the electrocapillary curves, and a line tension that decreases with the electrode potential, are valid in the absence of specific adsorption, since they are independent of the details of the model.

The authors gratefully acknowledge financial support by the Volkswagenstiftung (W. S.) and by the Fonds der Chemischen Industrie (H. I.), and useful discussions with Rolando Guidelli (Florence) and with Ansgar Liebsch. Critical reading of the manuscript by Margret Giesen and fruitful discussions are greatly appreciated.

*Corresponding author.

[1] E. B. Budewski, G.T. Staikov, and W. J. Lorenz, Electrochemical Phase Formation and Growth (WileyVCH, Weinheim, 1996).

[2] D. C. Schler, L. K. Verheij, G. Rosenfeld, and G. Comsa, Phys. Rev. Lett. 82, 3843 (1999).

[3] C. Steimer, M. Giesen, L. Verheij, and H. Ibach, Phys. Rev. B 64, 5416 (2001).

[4] S. Dieluweit, H. Ibach, and M. Giesen, R. Soc. Chem. Faraday Discuss. 121, 27 (2002).

[5] H. Ibach, M. Giesen, and W. Schmickler, J. Electroanal. Chem. 544, 13 (2003).

[6] W. Schmickler and D. Henderson, Prog. Surf. Sci. 22, 323-420 (1986); S. Amokrane and J. P. Badiali, Modern Aspects of Electrochemistry (Plenum Press, New York, 1992), Vol. 22.

[7] N. D. Lang and W. Kohn, Phys. Rev. B 1, 4555 (1970).

[8] N.W. Ashcroft and D.C. Langreth, Phys. Rev. 155, 682 (1967).

[9] W. Schmickler, Interfacial Electrochemistry (Oxford University Press, New York, 1996).

[10] W. Schmickler and E. Leiva, J. Electroanal. Chem. 453, 61 (1998).

[11] J. R. Smith, Phys. Rev. 181, 522 (1969).

[12] H. Ishida and A. Liebsch, Surf. Sci. 297, 106 (1993); Phys. Rev. B 46, 7153 (1992).

[13] M. D. Thompson and H. B. Huntington, Surf. Sci. 116, 522 (1982).

[14] R. Smoluchowski, Phys. Rev. 60, 661 (1941).

[15] K. Besocke, B. Krahl-Urban, and H. Wagner, Surf. Sci. 68, 39 (1977).

[16] M. Giesen, Prog. Surf. Sci. 68, 1 (2001). 